\title{
Entanglement and nonextensive statistics
}

\author{
A. Vidiella-Barranco* \\ Instituto de Física "Gleb Wataghin" \\ Universidade Estadual de Campinas \\ 13083-970 Campinas SP Brazil.
}

\begin{abstract}
It is presented a generalization of the von Neumann mutual information in the context of Tsallis' nonextensive statistics. As an example, entanglement between two (two-level) quantum subsystems is discussed. Important changes occur in the generalized mutual information, which measures the degree of entanglement, depending on the entropic index $q$.

Keywords: quantum information, entanglement, Tsallis statistics.
\end{abstract}

05.30.Ch; 03.67.-a; 89.70.+c

*Phone: +55 19 7885442; FAX:+55 19 7885427; e-mail:vidiella@ifi.unicamp.br 
Entropy is undoubtely one of the most important quantities in physics. After more than one century of its introduction, it still generates discussion around its nature and usefulness [1]. Of special importance it was the statistical interpretation of entropy given by Boltzmann, which allowed not only the development of classical statistical mechanics, but also the definition of its quantum mechanical counterpart, known as the von Neumann entropy [2]. The von Neumann entropy associated to a quantum state of a system described by a density operator $\hat{\rho}$ is

$$
S=-\operatorname{Tr}[\hat{\rho} \ln \hat{\rho}] \quad \operatorname{Tr} \hat{\rho}=1 .
$$

The von Neumann entropy does not depend on any of the system's observables, being a function of the state itself. It is easy to see that if the above mentioned system is in a pure state $\hat{\rho}=|\Psi\rangle\langle\Psi|$, its entropy vanishes. Moreover, under unitary evolution, entropy remains the same. However for statistical mixtures of pure states we have that $S>0$, i.e., classical uncertainties increase the entropy of the state.

Recently Tsallis [3] proposed a generalization of Boltzmann's entropy, which in the quantum mechanical case reads

$$
S_{q}=-\frac{1-\operatorname{Tr}\left[\hat{\rho}^{q}\right]}{1-q}
$$

The entropic index $q$ is a real parameter which is related to the (nonextensive) properties of the relevant physical system. In the limit of $q \rightarrow 1$, von Neumann's entropy is recovered. The Tsallis entropy has been succesfully applied to several interesting problems, involving nonextensive systems, which are normally untractable by means of Boltzmann's statistics. Amongst the problems treated within Tsallis' formalism we may cite the Lévy superdiffusion [4] and anomalous correlated diffusion [5], turbulence in 2D pure electron plasma [6], and in the analysis of the blackbody radiation [7]. There are more convenient values (or ranges of values) for the entropic index $q$, depending on the specific system being treated. For instance, in the problem of thermalization in electron-phonon systems, $q>1$ [8], while in the treatment of low dimensional dissipative systems, $q<1[9]$.

Entropy also plays a fundamental role in classical information theory 10 as well as in its quantum version. It may be considered as the average amount of information which is missing before observation. This may be quantitavely expressed, for instance, through the Kullback-Leibler measure of information [11], sometimes called relative entropy. A generalization of this measure and applications within Tsallis' statistics framework has been recently presented in the literature [12, 13, although in its classical version only. A discussion on channel capacities in nonextensive statistics may also be found in the literature [14]. Nevertheless there are normally not found in the literature discussions on the implications of generalized statistics in purely quantum mechanical problems, such as, for instance, on the measure of entanglement.

A quantity also used to compare distributions as well as quantum states is the mutual information or mutual entropy [15]. The quantum (von Neumann) mutual information $I$

\footnotetext{
${ }^{1}$ Boltzmann's constant $k$ has been set equal to one here.
} 
relative to two subsystems $(A$ and $B)$ may be written as

$$
I=S_{A}+S_{B}-S_{A B}
$$

where $S_{A}\left(S_{B}\right)$ is the entropy relative to the subsystem $A(B)$, and $S_{A B}$ is the entropy of the overall state, described by a density operator $\hat{\rho}_{A B}$. The reduced density operators relative to the subsystems, $\hat{\rho}_{A}$ and $\hat{\rho}_{B}$ are obtained from $\hat{\rho}_{A B}$ through the usual partial tracing operation, or

$$
\hat{\rho}_{A}=\operatorname{Tr}_{B} \hat{\rho}_{A B}, \quad \hat{\rho}_{B}=\operatorname{Tr}_{A} \hat{\rho}_{A B} .
$$

The von Neumann mutual information is then calculated using the quantum entropy in (四), i.e., $S_{i}=-\operatorname{Tr}\left[\hat{\rho}_{i} \ln \hat{\rho}_{i}\right]$. If the joint state $\hat{\rho}_{A B}$ is a pure state, then its von Neumann entropy $S_{A B}=0$, and according to the Araki-Lieb inequality [16]

$$
\left|S_{A}-S_{B}\right| \leq S_{A B} \leq S_{A}+S_{B}
$$

we have that $S_{A}=S_{B}$, which means that the von Neumann mutual information is simply $I=2 S_{A}$. In this pure state case, there are no classical uncertainties, and the correlations are purely quantum mechanical. Otherwise, if the joint state is a statistical mixture, $S_{A B}>0$, and there will be a mixing of classical and quantum correlations. A convenient property of the von Neumann mutual information is that it is always positive definite.

Quantum information theory has experienced a remarkable growth in the past years [17], mainly motivated by potential applications in communication and computation. In particular, the adequate measure of quantum correlations and entanglement [18] is of central importance in this field. It would be therefore interesting to discuss a measure of correlations such as the mutual information, in a more general context. Here I present a straightforward generalization of the von Neumann mutual information based on Tsallis entropy $\left(S_{q}\right)$, or

$$
I_{q}=S_{q A}+S_{q B}-S_{q A B}
$$

This quantity would represent a generalization of the measure of correlations for a wider class of quantum systems (nonextensive).

Now I am going to discuss an example involving a pair of two-state subsystems, $A$ and $B$. The relevevant basis states will be denoted as $\left|0_{\alpha}\right\rangle$ and $\left|1_{\alpha}\right\rangle(\alpha=A, B)$, and $\left\langle i_{\alpha} \mid j_{\alpha}\right\rangle=\delta_{i j}$. Let us assume that the overall system ( $A$ plus $\left.B\right)$ is prepared in a state represented by the following state vector:

$$
|\Psi\rangle_{A B}=p^{1 / 2}\left|0_{A}\right\rangle\left|1_{B}\right\rangle+(1-p)^{1 / 2}\left|0_{B}\right\rangle\left|1_{A}\right\rangle,
$$

with $0 \leq p \leq 1$. If $p=0.5$ we have a maximally entangled state, and for $p=0$ (or $p=1$ ) we have a disentangled (or product) state. We may even write a more general state, which could include loss of coherence, as

$$
\begin{aligned}
\hat{\rho}_{A B}= & p\left|0_{A}\right\rangle\left|1_{B}\right\rangle\left\langle 1_{B}\left|\left\langle 0_{A}|+(1-p)| 1_{A}\right\rangle\right| 0_{B}\right\rangle\left\langle 0_{B}\right|\left\langle 1_{A}\right|+ \\
& \gamma^{1 / 2}[p(1-p)]^{1 / 2}\left(\left|0_{A}\right\rangle\left|1_{B}\right\rangle\left\langle 0_{B}\left|\left\langle 1_{A}|+| 1_{A}\right\rangle\right| 0_{B}\right\rangle\left\langle 1_{B}\right|\left\langle 0_{A}\right|\right),
\end{aligned}
$$


where the parameter $\gamma(0 \leq \gamma \leq 1)$ determines whether the state $\hat{\rho}_{A B}$ is a pure entangled state $(\gamma=1)$, or a statistical mixture $(\gamma=0)$. The partial tracing operation (4) produces the following density operators relative to the subsystems $A$ and $B$

$$
\hat{\rho}_{A}=p\left|0_{A}\right\rangle\left\langle 0_{A}|+(1-p)| 1_{A}\right\rangle\left\langle 1_{A}\right|,
$$

and

$$
\hat{\rho}_{B}=(1-p)\left|0_{B}\right\rangle\left\langle 0_{B}|+p| 1_{B}\right\rangle\left\langle 1_{B}\right| .
$$

The generalized mutual information for this particular system will then read

$$
I_{q}=\frac{1}{q-1}\left[1+\eta_{+}^{q}+\eta_{-}^{q}-2\left(p^{q}+(1-p)^{q}\right)\right]
$$

where

$$
\eta_{ \pm}=\frac{1}{2}[1 \pm \sqrt{1+4 p(1-p)(1-\gamma)}]
$$

Now I analyze the behaviour of the generalized mutual information as a function of the entropic parameter $q$. I shall remark that in the global pure state case $(\gamma=1)$, the mutual information exactly represents the degree of entanglement between the states belonging to the subsystems $A$ and $B$, due to the lack of "classical noise". In Fig. 1 we have a plot of the generalized mutual information $I_{q}$ as a function of $q$ for different values of the parameter $\gamma$, and with $p=0.5$, which corresponds to a maximally entangled state in the case of $\hat{\rho}_{A B}$ being a pure state. For the range of values of $q$ here chosen $(0 \leq q \leq 2)$ the generalized mutual information is positive-definite 2 . If $q=1$ we have the usual von Neumann mutual information. For $\gamma=1$ (pure state), the mutual information decreases monotonically. It attains its maximum value, $I_{q}=2$, at $q=0$, and goes assimptotically to zero as $q$ increases. However, an interesting behaviour is noticeable for states having a small deviation from a pure state. For instance, if $\gamma=0.999$, although the von Neumann mutual information remains basically the same, $(I \approx 2 \ln 2)$, the generalized mutual information will substantially differ from that of a pure state $(\gamma=1.0)$ for not so large values of $q(q<0.5)$, as it is seen in Fig. 1. It starts increasing up to a maximum value at $q \approx 0.33$, then decreasing again. In this case there will be in general two different values of $q$ giving the same mutual information.

We may also analyze the behaviour of the generalized mutual information for a fixed $\gamma=1$ (pure state) for different values of the weight $p$. If $p=1$ the state is a pure disentangled one, having therefore mutual information $I_{q}$ equal to zero. However, even for a very small "entangled component", for instance, if we take $p=0.999$, the generalized mutual information will assume nonzero values for a range of values of $q$. This is shown in Fig. 2. For $q=1, I_{q} \approx 0$, which means that according to von Neumann's mutual information, the state is viewed as being completely disentangled. For other values of $q$, however, the generalized mutual information may be nonzero. This shows an extreme sensitivity of this measure of the degree of entanglement on the entropic index $q$, specially when the quantum state is very close of being either a pure state or an entangled state.

\footnotetext{
${ }^{2}$ Actually, $I_{q}$ is positive even for larger values of $q$.
} 
We conclude that entanglement may arise (or be enhanced), depending on the properties of a given physical system, such as extensivity, which is by its turn quantified by the entropic index $q$.

The definition for the generalized mutual information here presented $\left(I_{q}\right.$ in Eq. (6)) is not the only possible one. In fact, in [13] it is proposed, in its classical version, a slightly different form for a generalization of the mutual information. It would be therefore worth comparing our definition of mutual information in (6) with the quantum mechanical counterpart of the one found in reference [13], which may be written as

$$
\begin{aligned}
I_{q}^{\prime} & =S_{q A}+S_{q B}-S_{q A B}+(1-q) S_{q A} S_{q B}=I_{q}+(1-q) S_{q A} S_{q B} \\
& =\frac{1}{q-1}\left[1+\eta_{+}^{q}+\eta_{-}^{q}-2\left(p^{q}+(1-p)^{q}\right)-\left(1-p^{q}-(1-p)^{q}\right)^{2}\right] .
\end{aligned}
$$

We note that the definition of the generalized mutual information given above contains an additional "crossed term" $(1-q) S_{q A} S_{q B}$ relatively to the definition we have already used $\left(I_{q}\right)$. This might result in some differences, which of course will depend on the values of the parameters $p$ and $\gamma$. In the limit of $q \rightarrow 1$, von Neumann's mutual information is recovered in either case. It would be convenient to perform a graphical comparison. For that I have plotted in Fig. 3 the generalized mutual information $I_{q}^{\prime}$ from Eq.(13) as a function of the entropic index $q$ having $p=0.5$, analogously to the situation depicted in Fig. 1 . Despite of the differences we verify, both definitions of the generalized mutual entropy, $I_{q}$ and $I_{q}^{\prime}$ exhibit a very similar qualitative behaviour. In particular, the important sensitivity discussed above is present in both cases. The same is true in the case in which there is a small "entangled component" (analogous to the situation in Fig. 2). The corresponding plot for $I_{q}^{\prime}$ is shown in Fig. 4, and again, its qualitative behaviour is about the same as the one in Fig. 2. This means that the discussion carried out above is also valid in the case of the alternative generalized mutual information $I_{q}^{\prime}$. In summary, the "crossed term" $(1-q) S_{q A} S_{q B}$ does not appreciably affect the (qualitative) behaviour of the generalized mutual information for those values of the parameters $p$ and $\gamma$ which are relevant, at least for what has been discussed here.

I have presented a generalization of the quantum mechanical von Neumann's mutual information within Tsallis' nonextensive statistics. This observable-independent quantity, here denoted as $I_{q}$, is important for determining the degree of entanglement between different subsystems, for instance. I have found that depending on the value of the entropic index $q$ characteristic of Tsallis statistics, the generalized mutual information, which measures quantum correlations may assume very different values from those obtained in the von Neumann case $(q=1)$. The strong dependence of the mutual information on the entropic index may be of course associated to the extensivity properties of the relevant physical system, and entanglement arises for not so large values of $q$. I have also compared two different possible definitions of the generalized mutual information, showing that the sensitivity relatively to the entropic index $q$ is basically the same way in both cases. This represents a first attempt of establishing a connection between an intrinsic property of physical systems (extensivity) and the measure of the degree of entanglement between different susbsystems. 


\section{References}

[1] B. Wehrl, Rev. Mod. Phys. 50 (1978) 221.

[2] J. von Neumann, The Mathematical Foundations of Quantum Mechanics (Princeton University Press, Princeton, 1955).

[3] C. Tsallis, J. Stat. Phys. 52 (1988) 479. See also Nonextensive Statistical Mechanics and Thermodynamics, eds. S.R.A. Salinas and C. Tsallis, Braz. J. of Phys. 29 (1999).

[4] D.H. Zanette and Alemany, Phys. Rev. Lett. 75, 366 (1995); C. Tsallis, S.V.F. Levy, A.M.C. Souza, and R. Maynard, Phys. Rev. Lett. 75, 366 (1995).

[5] A.R. Plastino and A. Plastino, Phys. Lett. A 222 (1995) 347; C. Tsallis and D.J. Bukman Phys. Rev. E 54 (1996) R2197.

[6] B.M. Boghosian, Phys. Rev. E 53 (1996) 4754.

[7] C. Tsallis, F.C. Sá Barreto, E.D. Loh, Phys. Rev. E 52 (1995) 1447; Q.A. Wang and A.L. Mehaute, Phys. Lett. A 237 (1997) 28; E.K. Lenzi and R.S. Mendes, Phys. Lett. A 250 (1998) 270.

[8] I. Koponen, Phys. Rev. E 55 (1998) 7759.

[9] M.L. Lyra and C. Tsallis, Phys. Rev. Lett. 80 (1998) 53.

[10] C.E. Shannon and W. Weaver, The Mathematical Theory of Communication (Princeton University Press, Princeton, 1963).

[11] S. Kullback and R.A. Leibler, Ann. Math. Stat. 22 (1961) 79.

[12] C. Tsallis, Phys. Rev. E 58 (1998) 1442.

[13] L. Borland, A.R. Plastino and C. Tsallis, J. Math. Phys. 39 (1998) 6490; Erratum, J. Math. Phys. 40 (1999) 2196.

[14] P.T. Landsberg and V. Vedral, Phys. Lett. A 247 (1998) 211.

[15] M. Ohya, IEEE T Inform Theory 29 (1983) 770.

[16] H. Araki and E. Lieb, Commun. Math. Phys. 18 (1970) 160.

[17] A. Zeilinger, Rev. Mod. Phys. 71 (1999) S288.

[18] V. Vedral, M.B. Plenio, K. Jacobs, and P.L. Knight, Phys. Rev. A 56 (1997) 4452. 


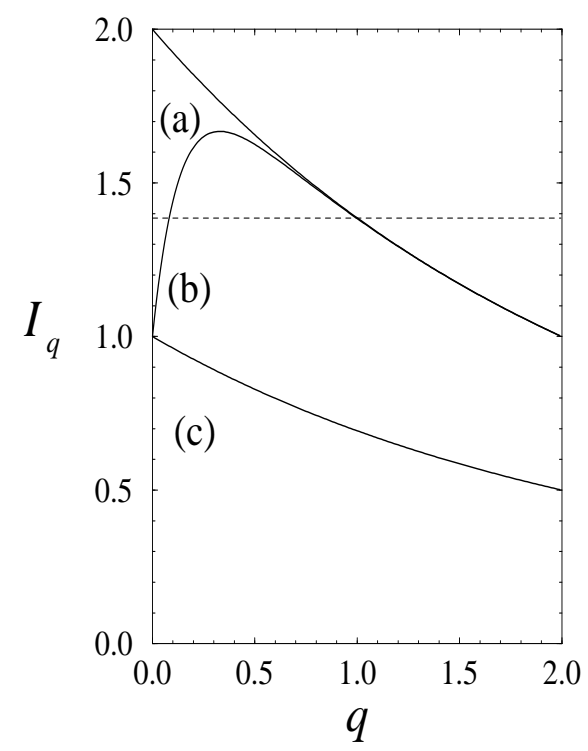

Figure 1: Generalized mutual information $I_{q}$ as a function of the entropic index $q$ for different values of $\gamma$ : (a) $\gamma=1$; (b) $\gamma=0.999$; (c) $\gamma=0$. In any case $p=0.5$. The dashed line indicates the von Neumann mutual information for the pure entangled state $I=2 \ln 2(\gamma=1)$.

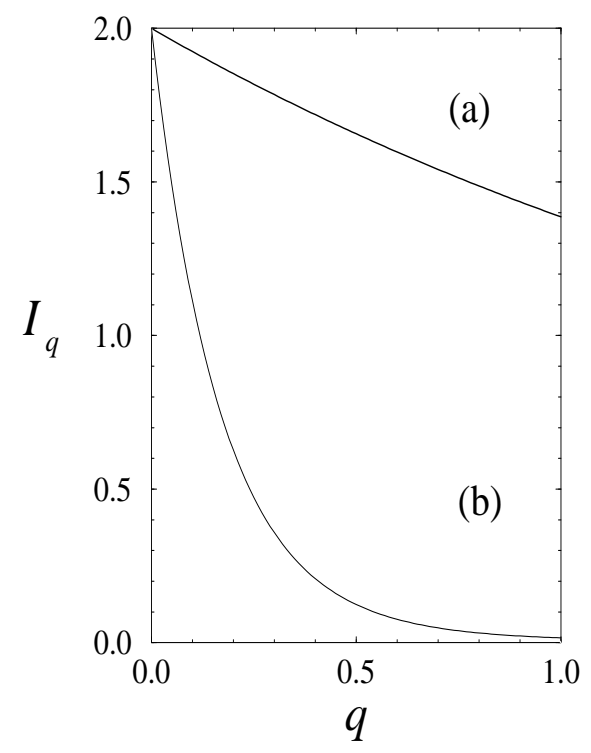

Figure 2: Generalized mutual information $I_{q}$ as a function of the entropic index $q$ in the pure state case $(\gamma=1)$ for (a) $p=0.5$; (b) $p=0.999$. 


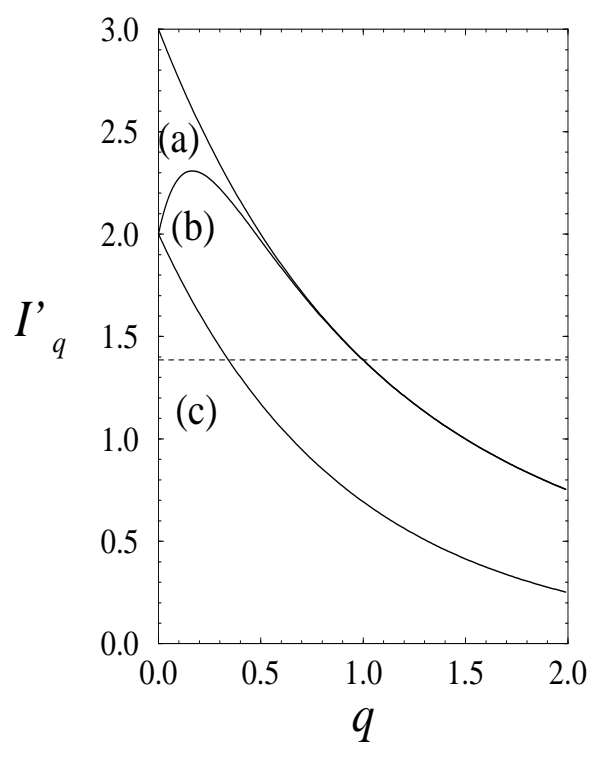

Figure 3: Alternative generalized mutual information $I_{q}^{\prime}$ as a function of the entropic index $q$ for different values of $\gamma$ : (a) $\gamma=1$; (b) $\gamma=0.999$; (c) $\gamma=0$. In any case $p=0.5$. The dashed line indicates the von Neumann mutual information for the pure entangled state $I=2 \ln 2(\gamma=1)$.

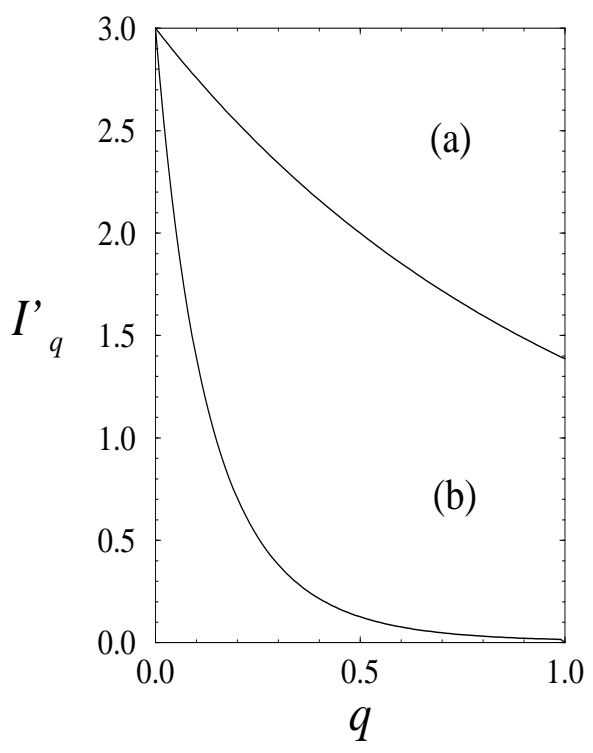

Figure 4: Alternative generalized mutual information $I_{q}^{\prime}$ as a function of the entropic index $q$ in the pure state case $(\gamma=1)$ for (a) $p=0.5$; (b) $p=0.999$. 